\title{
Fast ion-selective ppb-level gas sensor based on pulsed atmospheric pressure chemical ionization and ion-ion- recombination
}

\author{
A. Heptner ${ }^{1}$, N. Angerstein ${ }^{1}$, S. Zimmermann ${ }^{1}$ \\ ${ }^{1}$ Leibniz University Hannover, Institute of Electrical Engineering and Measurement Technology, \\ Department of Sensors and Measurement Technology, Appelstr. 9A, 30167 Hannover, Germany \\ e-mail: heptner@geml.uni-hannover.de
}

\begin{abstract}
Investigating the ion-ion-recombination rate of different analytes at atmospheric pressure reveals that ion species can be separated by their ion specific recombination rates. This effect is utilized for the development of a novel gas sensor: A gas mixture is ionized by atmospheric pressure chemical ionization (APCl) for a defined ionization time. At the end of the ionization the generated ion species recombine leading to a decreasing ion density with an increasing recombination time. The measured ion density at different times leads to characteristic decay curves depending on the ion species present and thus on the gas composition. From this decay curves analytes can be identified and analyte concentrations down to ppb-levels can be detected.
\end{abstract}

Key words: atmospheric pressure chemical ionization, ion-ion-recombination, ion specific recombination rates, known measurement environment

\section{Introduction}

Gas detection is an important sector in the measurement technology. For example it is desired to detect chemical warfare agents, toxic industrial compounds, explosives and drugs of abuse [1-4]. Therefore, the measurement systems have to be very selective to secure an exact identification of the compounds in an unknown gas mixture. Because even small amounts of hazardous compounds can have a high impact on the human health one requirement is the very high sensitivity of low concentrations up to parts-per-billion (ppb). For example a mass spectrometer (MS) can be used to analyze unknown gas mixtures. Here, the compounds are ionized by an ionization source and the generated ion species are separated by their mass to charge ratio which leads to a mass spectrum with intensity over the mass to charge ratio. This technology is very selective and sensitive, however, the instrumental effort is very complex and expensive. Furthermore, an ion mobility spectrometer (IMS) can be used for the analysis of gas mixtures [5]. Here, the compounds are ionized and then separated by their ion specific ion mobility. Thus, an ion mobility spectrum is generated with signal intensity over the ion mobility. In contrast to mass spectrometer IMS operates at ambient pressure. The drawback of an IMS compared to a MS is the lower resolving power. However, while the sensitivity is still in the ppb-level the instrumental effort and costs are much lower enabling online monitoring and field applications.

For the monitoring of known measurement environments sensors with less analytical performance can be used as well at lower effort. In this work we present a sensor principle based on atmospheric pressure chemical ionization (APCI) with a sensitivity in the ppb-level [6]. As separation technique the time dependent and ion specific ion-ionrecombination kinetics is used [7]. Due to the ion specific recombination rates the compounds of gas mixtures on several analyte concentrations can be distinguished [8]. In this paper we describe the atmospheric pressure chemical ionization method, the theory of ionion-recombination, the construction of the simple gas detector and the used measurement equipment. Further, a generated sammon mapping [9] for pattern recognition for the measured decay curves is shown. 


\section{Fundamentals}

In our work a pulsable non-radioactive electron gun as ionization source is used. This electron gun emits high kinetic electrons into the ionization region for a definable time. Due to the high concentration of nitrogen in air mostly nitrogen ions are generated. According to the reaction chain which is described by [10] the positive $\mathrm{H}^{+}\left(\mathrm{H}_{2} \mathrm{O}\right)_{n}$ and negative $\mathrm{O}_{2}-\left(\mathrm{H}_{2} \mathrm{O}\right)_{n}$ reactant ions are formed. In a next step analyte ions are generated by charge transfer reaction according to eq. 1. The requirement for generating positive analyte ions is the higher proton affinity of the analyte molecules in relation to the proton affinity of the positive reactant ions. It is important to know that one analyte can build several ion species. For example acetone generates monomer ion species which consists of one acetone molecule and dimer ion species which consists of two acetone molecules.

$$
A+H^{+}\left(\mathrm{H}_{2} \mathrm{O}\right)_{n} \rightleftarrows \mathrm{AH}^{+}\left(\mathrm{H}_{2} \mathrm{O}\right)_{n-1}+\mathrm{H}_{2} \mathrm{O}
$$

Analogues to the generation of positive analyte ions the generation of negative analyte ions depends on the higher electron affinity in relation to the negative reactant ions.

There are many ion loss mechanism in the ionization region, e.g. ions can diffuse to metallic surfaces and discharge. However, in this application ion-ion-recombination is the major ion loss mechanism [7]. The neutralization of only one positive and one negative ion species with each other in a macroscopic field free and ion filled volume can be described by eq. 2. The decay of positive and negative ions per time depends on the ion concentrations $N_{+}$and $N$. of the involved recombination partner and the ion specific recombination rate $k_{r}$ which depends on the involved recombination partner as well.

$$
\frac{d N_{+}}{d t}=\frac{d N_{-}}{d t}=-k_{r} \cdot N_{+} \cdot N_{-}+S
$$

Assuming that the ion generation is turned off the ion production rate $S$ becomes zero and no additional ions are generated. By using high kinetic electrons for ionization the equal amount of positive and negative ions $N_{+}=N$ - is assumed [11]. Thus, eq. 2 is simplified to eq. 3 .

$$
\frac{d N_{+}}{d t}=\frac{d N_{-}}{d t}=-k_{r} \cdot N^{2}
$$

In addition, only singly ionized ion species are assumed [7]. Thus, eq. 3 can be solved and eq. 4 is received. The charge $Q\left(t_{\text {rec }}\right)$ depends on the initial charge $Q_{0}$ the recombination time $t_{r e c}$ and the ion specific recombination rate $k_{r}$.

$\left|Q\left(t_{r e c}\right)^{-1}\right|=\frac{1}{\left|Q_{0}\right|}+k_{r} \cdot t_{\text {rec }}$

In presence of only one positive and one negative ion species the decay curve equals a straight line with the slope $k_{r}$. Thus, different positive ion species recombining with the negative reactant ions can be clearly distinguished by calculating the slope of the measured decay curves [8]. As shown in [12] the initial charge $Q_{0}$ has an influence on the recombination rate $k_{r}$, which is shown in eq. 5 . For a clearly comparison of the recombination rates of different ion species the initial charge has to be kept constant.

$k_{r}=k_{r t}+\frac{C}{Q_{0}}$

For the investigation of ion-ion-recombination it is important to use an ionization source which can be turned on and off. In addition the emission current has to be adjustable which secure reproducible measurements and constant $Q_{0}$.

\section{Experimental section}

This sensor which is shown in Fig. 1 mainly consists of a pulsable ionization source (1) [13], a reaction chamber (2) including a faraday detector (3), a transimpedance amplifier (4), a push-electrode (5) and an electronic control and processing unit (6).

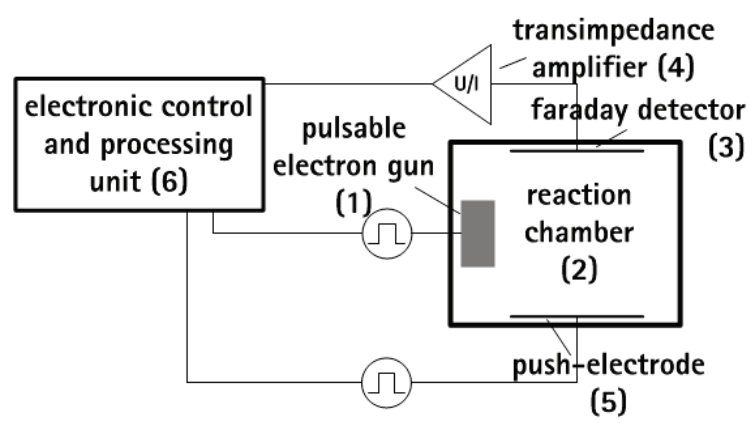

Fig. 1: Schematic of the ion-selective gas sensor consisting of a pulsed ionization source (1), the reaction chamber(2), a faraday detector (3), a transimpedance amplifier (4), a push electrode (5), and the electric control system (6).

The used electron gun emits a definable amount of electrons with a kinetic energy of approximately $8.8 \mathrm{keV}$ into the reaction 
chamber. These emitted electrons ionize the neutral gas mixture for a defined time. The assumption of an equal amount of positive and negative ions for solving eq. 3 to eq. 4 is fulfilled due to one high kinetic electron generating nearly 600 cations and 601 anions. In Fig. 2 the ion filled reaction chamber at the end of the electron emission is shown.

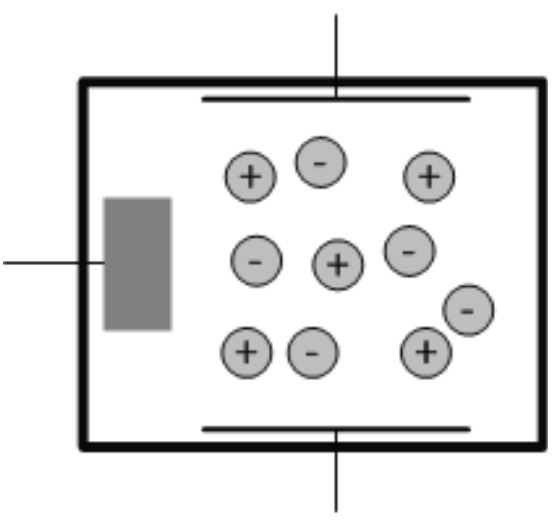

Fig. 2: Schematic of the ion filled reaction chamber at the end of the defined ionization time with equal amount of singly ionized positive and negative ions.

For the investiagtion of the ion-ionrecombiantion two requirements have to be secured. On the one hand the ionization source has to be turned off entailing to no additional ion generation. On the other hand the reaction chamber has to be macroscopic electrical field free which requires equal electrical potentials at all metallic surfaces in the reaction chamber during the recombination time. Otherweise an electrical field would separate the positive and negative ions leading to a discharge at metallic surfaces. Fig. 3 shows the decreased ion density in the reaction chamber at the end of a defined recombination time $t_{\text {rec. }}$. Here, the long-living ion species are still present in the reaction chamber while the short-living ion species are neutralized.

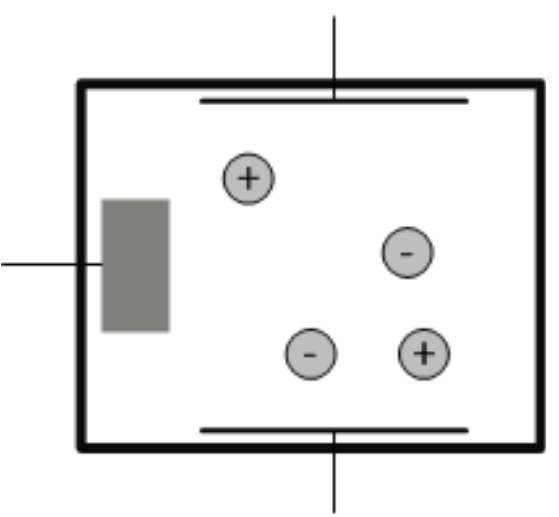

Fig. 3: After a defined recombination time the ion density in the reaction chamber is decreased. The long lived ion species are still present.

To stop the ion-ion-recombination at a defined recombination time a transient electrical field is applied between the faraday detector and the push-electrode. Thus, ions of one polarity discharge at the push-electrode while the ions of the other polarity discharge at the faraday detector generating an ion current (see Fig. 4).

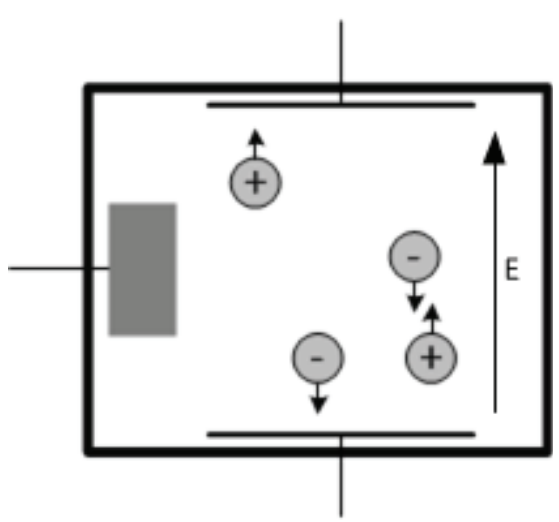

Fig. 4: After the defined recombination time the ions are separated by a transient electrical field. Thus the ion current of one polarity can be measured.

Assuming that only singly ionized ion species are generated [7] the ion density is proportional to the ion current. The integration of this ion current over time equals to the total charge in the reaction chamber. The determination of the charge at different recombination times leads to a decay curve of this total ion charge $Q\left(t_{\text {rec }}\right)$. The ion-ion-recombination kinetics are characteristic for the recombining ion species. Therefore, the decay curves are characteristic for the compounds in a gas mixture.

The investigated gas mixtures are generated by mixing dry clean air, moist gas and analyte gas which is adjusted by a permation oven. Thus, we can configure the relative humidity $r_{H}$ and the analyte concentration very exactly. In addition a dew point sensor to monitore the relative humidity is used.

\section{Results and discussion}

lonizing clean air with a relative humidity of $40 \%$ generates $\mathrm{H}^{+}\left(\mathrm{H}_{2} \mathrm{O}\right)_{n}$ and $\mathrm{O}_{2}-\left(\mathrm{H}_{2} \mathrm{O}\right)_{n}$ ions. According to the theory of ion-ionrecombination (eq. 3) the decay curve (solid line in Fig. 5) of only one positive and one negative ion species equals a straight line [8]. However, ionizing the analyte dimethylmethylphosphonate (DMMP) in air generates monomer and dimer ions of DMMP. Increasing the DMMP concentration results in an increasing deviation of the decay curves compared to the decay curve of clean air as can be seen in Fig. 5. Therefore, the DMMP concentration can be derived from virtually any point in the decay curve. 


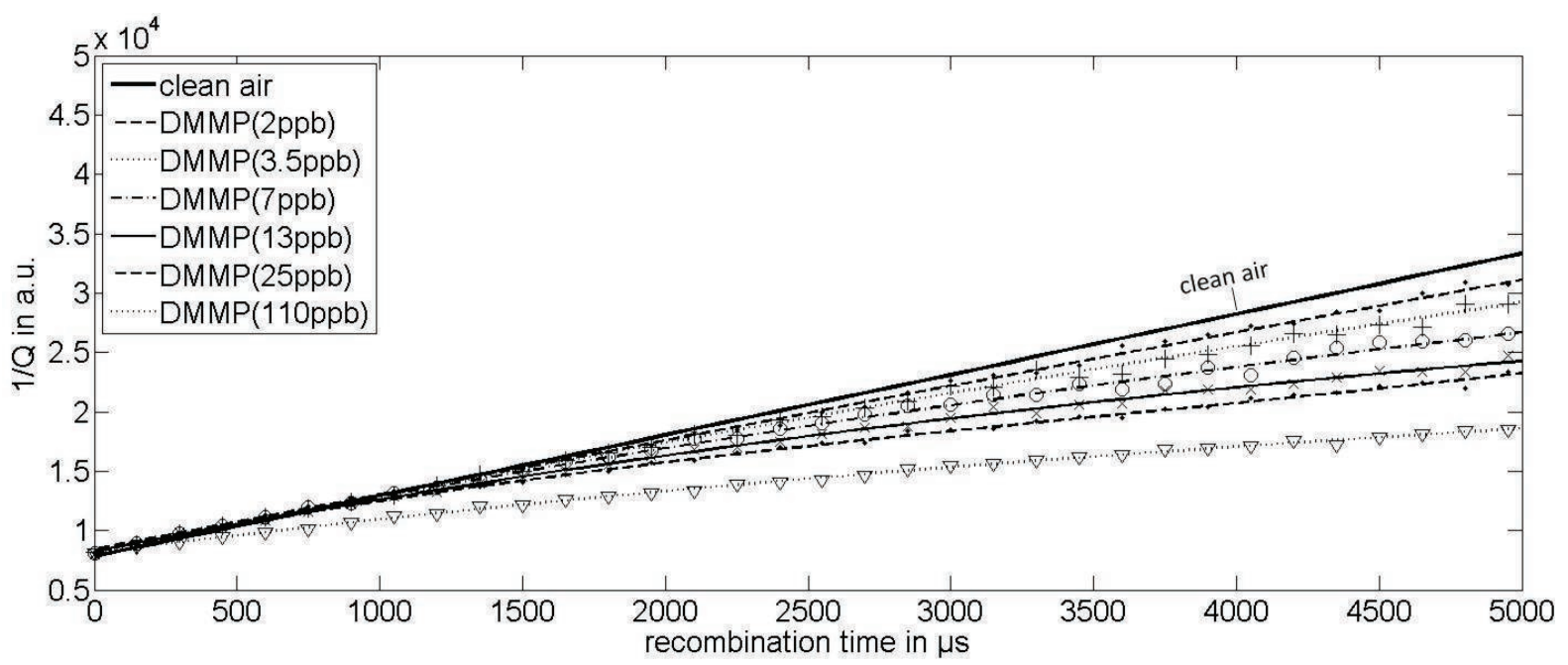

Fig. 5: Decay curves of clean air containing different concentrations of dimethyl-methylphosphonate. With increasing concentration of DMMP the slope of the decay curves decrease and the deviation to the decay curve of clean air increase.

As another example, the effect of ammonia ions on the ion-ion-recombination and the measured decay curve is investigated, see Fig. 6. As for DMMP, increasing the ammonia concentration in air results in an increasing deviation from the decay curve of clean air. It can be seen that the total charge of the measurement with ammonia is lower than the total charge of DMMP at same recombination times. With this examples we show the possibility to generally distinguish different analytes by only considering the ionion-recombination kinetics.

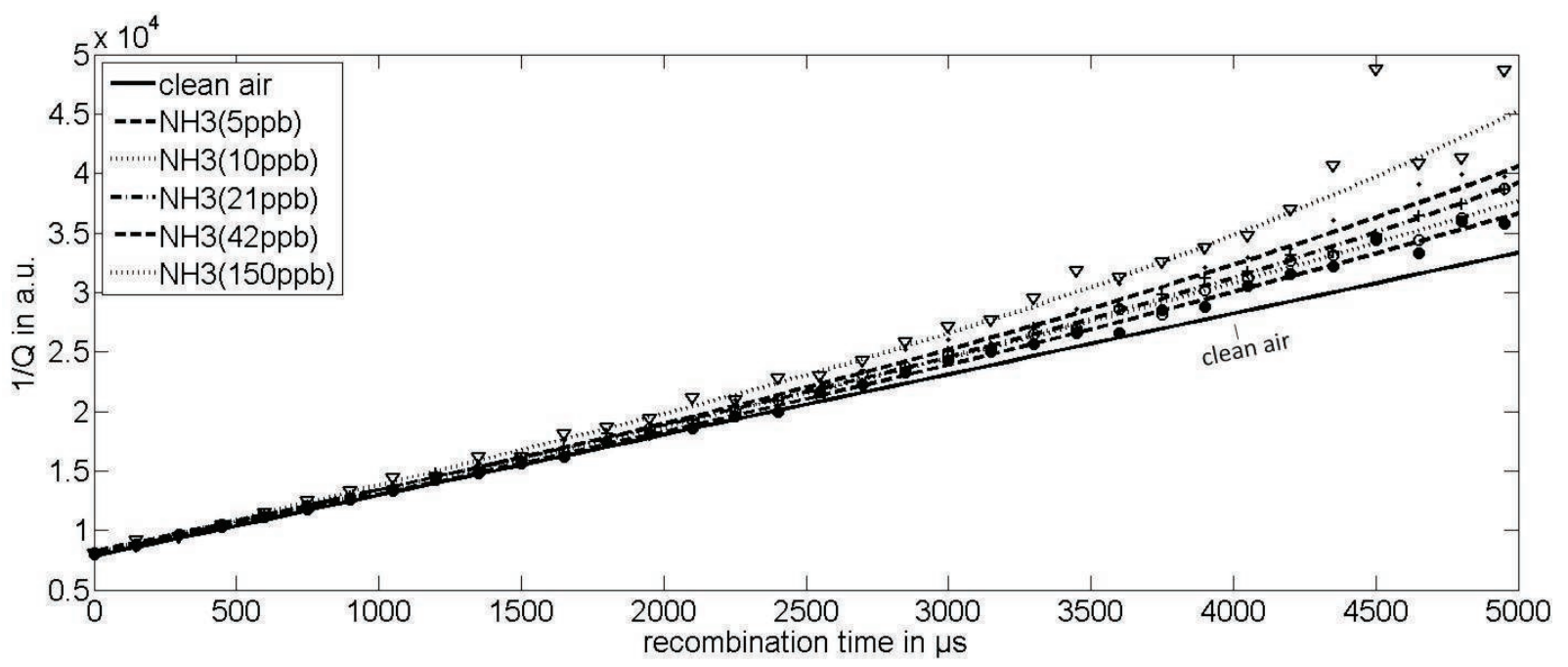

Fig. 6: Decay curves of clean air containing different concentrations of ammonia. With increasing concentration of ammonia the slope of the decay curves increase and the deviation to the decay curve of clean air increase too.

In a next step gas mixtures of ammonia and DMMP by measuring decay curves are investigated. Here, we measured decay curves at six different DMMP concentrations by varying three ammonia concentrations which generates 18 decay curves. As can be seen in Fig. 7 the characteristic decay curves compared to the decay curves of only ammonia or DMMP are more complex. Only considering the total charge in the reaction chamber at one recombination time can lead to a misinterpretation of the existing gas compounds. As shown in Fig. 7 the progress of a decay curve of the gas mixture can change. Until a recombination time of $t_{\text {rec }}=3.6 \mathrm{~ms}$ the decay curves of the gas mixture containing 20 ppb ammonia and 150 ppb DMMP and the gas mixture containing $10 \mathrm{ppb}$ ammonia and $40 \mathrm{ppb}$ DMMP are similar to the decay curves of the measurements with only DMMP as analyte. After $t_{\text {rec }}=3.6 \mathrm{~ms}$ the progress of the decay curves are similar to the decay curves with only 
ammonia as analyte. In addition, at $t_{r e c}=3.6 \mathrm{~ms}$ the total charge equals the total charge of clean air. This observation could lead to the assumption that no analyte is present in the investigated gas mixture. However, considering only one point of the decay curves is not sufficient. The whole decay curve has to be considered.

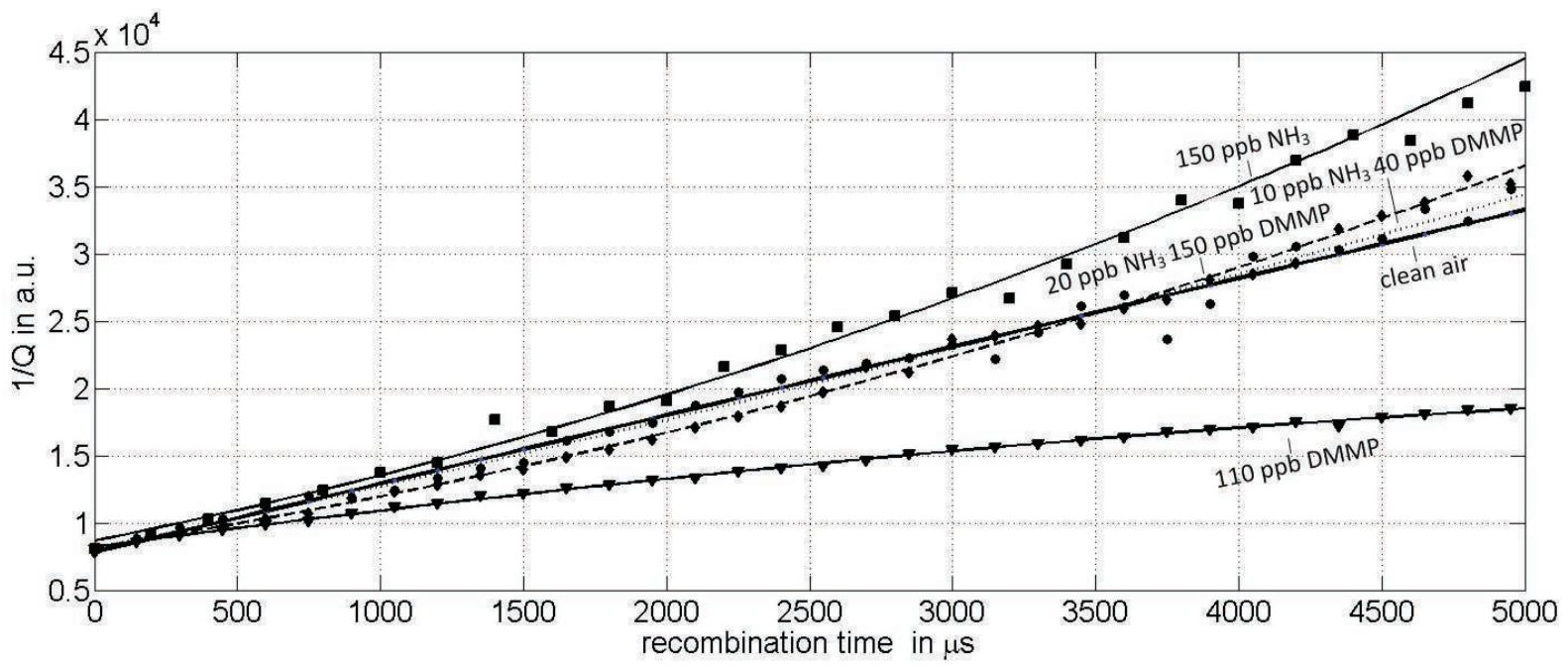

Fig. 7: Decay curves of clean air containing different concentrations of ammonia and DMMP. Changing the analyte concentrations leads to different characteristic decay curves.

As shown in Fig. 7 the comparison of the decay curves is very complex. Therefore, we use pattern recognition to visualize the measurement data. In the following, each decay curve is represented by the measured ion concentration at $\mathrm{n}=10$ defined equidistant recombination times giving one $n$-dimensional vector for each decay curve. Every decay curve is reduced to one point in the n-dimensional space by using the sammon algorithm. To visualize the distribution of all measured decay curves the sammon mapping is shown in Fig. 8 [9]. As can be seen in Fig. 8, even low ppb-level concentrations of both DMMP and ammonia can be clearly distinguished from clean air and from each other. The analysis of the calculated points of the measurements with ammonia and DMMP as gas mixture is still complex. However, it is possible to classify the measurement points to realms of same DMMP concentrations by using the sammon algorithm. For example, the points with a concentration of 100 and 40 ppb DMMP by various ammonia concentrations generate compact realms in the sammon map. These points can be distinguished to the point of dry clean air as well. However, the reduction to one point for each decay curve leads to a more simple analysis of the sensor signal.

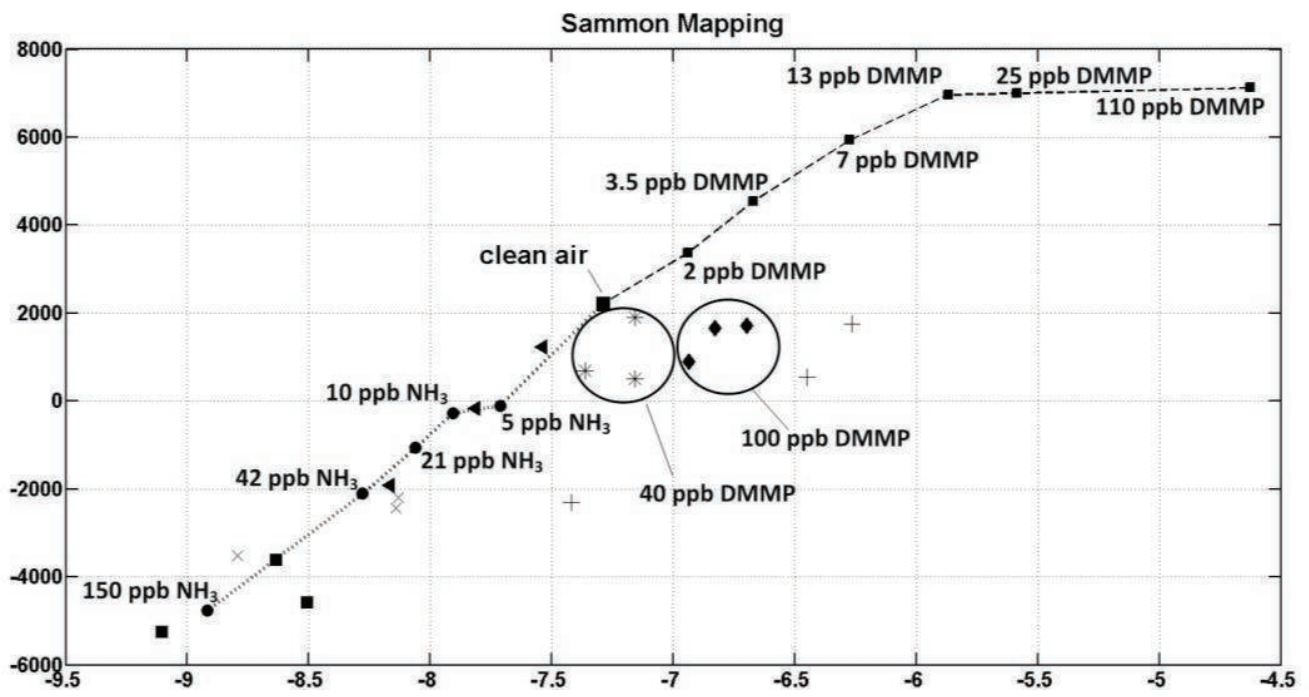

Fig. 8: Sammon map of decay curves with clean air containing DMMP, ammonia and gas mixtures containing DMMP and ammonia. 


\section{Conclusion}

In this paper we present the fundamentals of a novel ion-selective gas sensor. Due to the ionization mechanism of atmospheric pressure chemical ionization (APCl) this sensor is very sensitive at the ppb-level. The separation technique and the selectivity of this sensor is based on the ion-ion-recombination kinetics of an ion filled reaction chamber. For the measurement setup, a non-radioactive pulsable electron gun is used. Thus, the ion-ionrecombination kinetics in a macroscopically electrical field free reaction chamber can be investigated without the generation of additional ions. Considering our measurement data it can be seen that different concentrations of single analytes in clean air can be distinguished very well. In addition we used pattern recognition, the sammon algorithm respectively, to reduce the measurement data of one decay curve to one point in the sammon map. Here, the different concentrations of single analytes in clean air can be distinguished very well too.

We further measured the decay curves of gas mixtures containing different concentrations of dimethyl-methylphosphonate and ammonia. Considering the decay curves of these data is very complex. Further, the visualization with the sammon algorithm is used. To make a clear statement by considering the sammon map is not simple. However, for process monitoring of a known measurement environment this sensor principle can be used. A change of the analyte concentrations can be detected by the deviation to the training points.

In a next step the ion generation kinetics and their impact on the ion-ion-recombination kinetics will be investigated. Due to the additional information of the ion generation kinetics the resolving power of this novel ionselective sensor can be enhanced.

\section{Acknowledgements}

This research is funded by the German Research Foundation (DFG).

\section{References}

[1] Wes E. Steiner, B. H. Clowers, P. E. Haigh, H. H. Hill Jr., Secondary lonization of Chemical Warfare AgentSimulants: Atmospheric Pressure Ion MobilityTime-of-Flight Mass Spectrometry Anal. Chem. 75, 6068-6076(2003)

[2] J. E. Roehl, Environmental and Process Applications for Ion Mobility Spectrometry, Appl. Spectrosc. Rev. 26,1-57 (1991)
[3] C. Wu, W. E. Steiner, P. S. Tornatore, L. M. Matz, W. F. Siems, D. A. Atkinson, H. H. Hill Jr., Construction and characterization of a highflow, high-resolution ion mobility spectrometer for detection ofexplosives after personnel portal sampling Talanta 57, 123-134(2002);

[4] D. J. Weston, R. Bateman, Ian D. Wilson, Tim R. Wood, C. S. Creaser, Direct Analysis of Pharmaceutical Drug Formulations Using Ion Mobility Spectrometry/Quadrupole-Time-of-Flight Mass Spectrometry Combined with Desorption Electrospray Ionization, Anal. Chem. 77,75727580 (2005)

[5] H. Borsdorf, G. A. Eiceman, Ion Mobility Spectrometry: Principles and Applications, Appl. Spectrosc. Rev. , 41, 323-375 (2006)

[6] H. Borsdorf, E. G. Nazarov, G. A. Eiceman, Atmospheric pressure chemical ionization studies of non-polar isomeric hydrocarbons using ion mobility spectrometry and mass spectrometry with different ionization techniques, J. Am. Soc. Mass. Spec., 13, 9, 1078-1087 (2002)

[7] L. B. Loeb, Basic process of gasous electronics, Univ. of California Press (1960)

[8] A. Heptner, P. Cochems, J. Langejuergen, F. Gunzer, S. Zimmermann, Investigation of ion-ionrecombination at atmospheric pressure with a pulsed electron gun, Analyst 137, 21, 5105-5112 (2012)

[9] J. W. Sammon Jr., A Nonlinear Mapping for Data Structure Analysis, IEEE Trans. on Computers, C18, 5, 401-409 (1969)

[10] G. A. Eiceman, Z. Karpas, H. H. Hill Jr., Ion Mobility Spectrometry, CRC Press (2014)

[11] S. McGowan, Ion-Ion Recombination in Laboratory Air, Phys. Med. Biol., 10, 25-40 (1965)

[12] H. Jungblut, D. Hansen, W. F. Schmidt, Ion-ion Recombination in Electronegative Gases, IEEE Trans. Electr. Ins., 24, 2, 343-348 (1989)

[13] P. Cochems, M. Runge, S. Zimmermann, A current controlled miniaturized non-radioactive electron emitter for atmospheric pressure chemical ionization based on thermionic emission, Sensors and Actuators A, 206, 165-170 (2014) 